\title{
Biodiversity in Food Consumption Studies
}

\author{
Michelle Cristine Medeiros Jacob, Severina Carla Vieira Cunha Lima, \\ Clélia de Oliveira Lyra, and Dirce Maria Lobo Marchioni
}

\begin{tabular}{|c|c|c|}
\hline \multicolumn{3}{|c|}{ Contents } \\
\hline 1 & 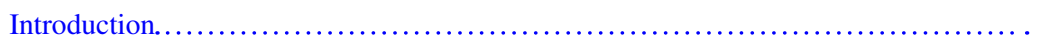 & 327 \\
\hline \multirow[t]{2}{*}{2} & An Overview of the Challenges Involved in Biodiverse Food Plants' Dietary & \\
\hline & Assessment & 328 \\
\hline 3 & Assessing Biodiverse Food Plants in Dietary Intake Surveys: How to Do It?.... & \\
\hline & Fundamental Actions to Mainstream Biodiverse Food Plants in Dietary & \\
\hline & 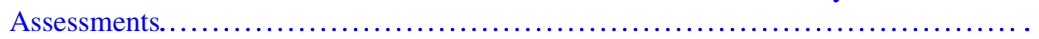 & \\
\hline & Conclusion.................................. & \\
\hline & ferences.................................. & \\
\hline
\end{tabular}

\section{Introduction}

In the last few decades, we have seen a tendency toward homogenization in human diets (Khoury et al. 2014). Diets that used to be composed of a wide variety of plants and animals have gradually shifted to include a limited number of species, focusing on very few crops and breeds. It is remarkable that while an estimated 30,000 edible plant species are available to humans, more than half of global food energy is currently met by only four crops: rice, potatoes, wheat, and maize (FAO 2010).

This tendency toward homogenization is problematic since low biodiversity can negatively impact human and environmental health (Willett et al. 2019). Broad evidence shows that food systems which neglect locally available nutrient-rich foods face the effects of simplified energy-dense diets, various forms of malnutrition, and a growing incidence of non-communicable diseases (Khoury et al. 2014; Herforth and Ahmed 2015). Agricultural biodiversity also increases food production resilience, especially in the face of sudden climate changes, disease outbreaks, and

\footnotetext{
M. C. M. Jacob $(\bowtie) \cdot$ S. C. V. C. Lima · C. de Oliveira Lyra

Department of Nutrition, Federal University of Rio Grande do Norte, Natal, Brazil e-mail: michellejacob@ufrn.edu.br

D. M. L. Marchioni

Department of Nutrition, Faculty of Public Health, University of São Paulo, São Paulo, Brazil e-mail: marchioni@usp.br
} 
market price fluctuations (Zimmerer 2015). Wild plant diversity, such as in unconventional food plants, is a strong component of food sustainability in many communities worldwide, especially during drought (Powell et al. 2015). The proper management and use of biodiversity can help to restore ecosystems and assist in resolving diet problems, such as micronutrient deficiencies in vulnerable populations (Ruel and Alderman 2013). However, it is worth mentioning that dietary and ecological indicators to assess the complex relationship between food biodiversity and quality of the diet must improve to provide greater accuracy (Lachat et al. 2018).

When assessing the impact of diet on human and environmental health, analysis of what people eat is crucial. In this chapter, our focus is on biodiverse food plants. The definition of biodiverse food plants (BFP) involves the agricultural diversity of conventional crops cultivated locally (e.g., varieties of beans, rice, corn, banana), together with unconventional food plants (UFP), (usually native, neglected, and rarely available in the marketplace) (Jacob and Albuquerque 2020). Despite the potential of these plants to promote sustainable food systems and diets, when we review the literature in dietary assessment studies, we notice a gap in methods for gathering and assessing the intake of these plants. This information gap is even more evident in studies developed for food systems with higher degrees of complexity (e.g., urban).

In this paper, we discuss certain challenges in assessing biodiverse food plants in dietary assessment and present (how to) proposals for step-by-step development. Finally, we summarize the actions needed to bring biodiverse food plants in dietary assessments mainstream. We hope that the debate concerning these limitations can help us to understand the real dietary contribution of these plants to the sustainability of the greater food system.

\section{An Overview of the Challenges Involved in Biodiverse Food Plants' Dietary Assessment}

To evaluate diet in free-living human beings is a complex task. Incorporating food biodiversity into dietary assessments creates an additional layer of complexity. Through field research, studying people of all life cycles, using the most varied dietary assessments, and analyzing the available literature, we noted the challenges seen in distinct phases of food composition studies that focus on biodiversity. In this topic, we will address these challenges in the three stages of dietary assessments: definition of goals, study design, and analysis.

A first critical step is to define the dietary assessment objectives. Usually, the dietary survey participants, especially in urban settings, are unable to even recognize the different varieties of vegetables they consume. In this sense, including photos of different food items might help the participants to accurately report the food they consume (FAO 2017). A photo book (or other kind of visual aid) containing food (and its varieties of interest), with different local names, might be a useful dietary assessment tool (in the next section, we will explore this in more detail). 
However, to map biodiversity is not simple. For this reason, in determining the extent of information on biodiverse foods which might be included in this visual tool, the assessment objectives must be clear. The team should develop a priority list based on the criteria set forming the survey's objectives.

As an example, we bring a pilot study that was performed in ISA research (Inquérito de Saúde de São Paulo, in Portuguese) in 2013, where our goal was to assess the diversity of food intake in São Paulo. In this study, we verified that the population presented dietary inadequacy for vitamin $\mathrm{A}$, vitamin $\mathrm{C}$, iron, and folate. Therefore, these were our target nutrients. By consulting the data of a previous survey, we listed all of the fruits, legumes, and vegetables that contribute to the intake of these nutrients consumed by the target population. Subsequently, we crossed the list with data supplied by the Ceagesp warehouse, the biggest supplier of these food items in the city, to identify the species in at least three different cultivars (see Fig. 1).

A second principal step is to define the study's layout. For this task, there are currently a variety of manuals and guidelines on dietary assessment available. However, these same manuals highlight their own cross-cultural limits and the need for personnel with professional capacity in biodiverse food plants, culture, and eating patterns (FAO 2018). In a nutshell, current dietary assessment methods present limits in approaching the nutritional contribution of biodiverse food in diets (FAO 2017). Diets themselves embed cultural and environmental factors. Analysts of diets with a focus on biodiversity must be doubly aware of this. Biodiversity is linked to cultural practices and the given landscape (Kuhnlein 2014). In the same way that ethnography helps to build contextual understanding about a people and their culture, it can support us in building a more robust dietary assessment design (see, e.g., Tumilowicz et al. (2016)).
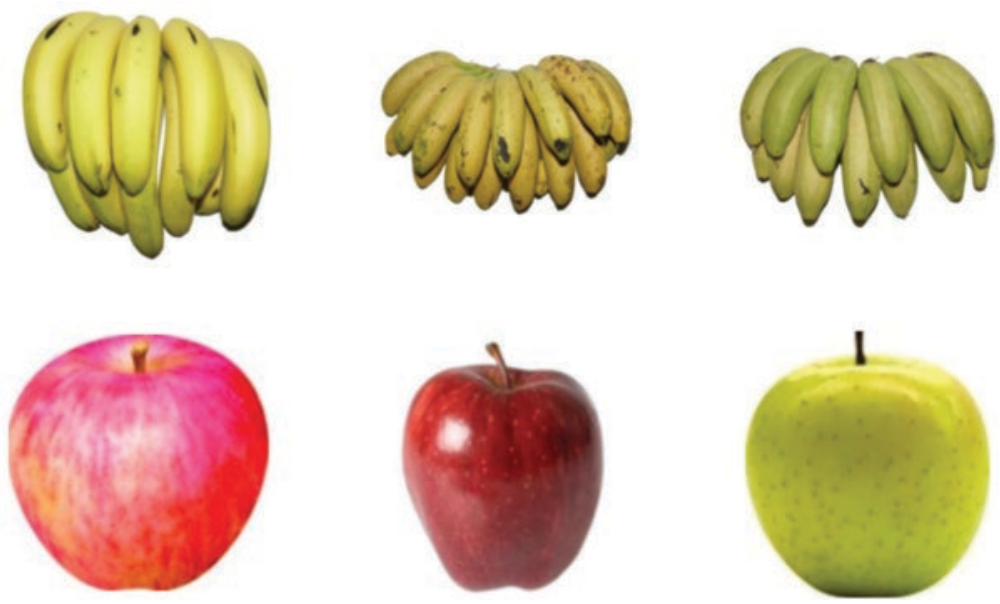

Fig. 1 Example of three varieties of bananas (Musa acuminata Colla x Musa balbisiana Colla) and apples (Malus domestica Borkh.) Available at the Ceagesp market in São Paulo. From top to bottom, left to right: banana-nanica, banana-ouro, banana-maçã, and maçã-gala, maçã-reddelicious, and maçã-golden. (Source: Ceagesp 2020) 
Here we note that an ethnographic diagnosis, when focused on food and nutrition, is an ethnonutritional assessment. Ethnonutrition is the study of diets in the context of food systems of differing peoples and cultures. An ethnonutritional assessment functions as a useful way to rapidly calibrate tools and prototype dietary assessments while considering cultural and environmental peculiarities of the local food system (Jacob and Albuquerque 2020). For example, in a classic ethnonutrition study in Cameroon, de Garine (1972) shows us how dishes and table utensils influence eating practices. People in many traditional societies often eat together from a typical dish, and the amount of food eaten by each person varies according to sex, age, or other situations. Thus, an ethnonutrition assessment can help us to define the best strategy for data-gathering and help to prepare domestic utensil manuals and inventory meals and beverages that are part of local culture. It is also important to identify local culinary processing techniques that may produce nutritional modulation in food composition and nutrient bioavailability. For example, when reviewing studies concerning BFP in the Brazilian Caatinga, one of us identified local consumption of an UFP (mucunã), considered toxic because of chemical composition studies (Grant et al. 1986), (Dioclea grandiflora Mart. ex Benth) (Jacob et al. 2020). Mucunã seeds likely contain small molecular weight components that cause its toxicity and which can be eliminated through exhaustive dialysis (Grant et al. 1986). In the community we studied, people empirically knew this. For this reason, they performed specific culinary processing, washing the flour several times (they use the expression in Portuguese lavar em várias águas) and eliminating the plant's toxins. By revealing the contextual culinary processing, previous ethnonutrition assessment permitted us to avoid misinterpretations that might lead to wrong conclusions in the dietary assessment.

The third step is analysis. Data on biodiverse food plants are scarce (FAO 2017), especially for plants identified below the species level (e.g., variety, cultivar). This lack of information is problematic, because when considering composition data for a specific variety, it promotes misinterpretation of the nutritional contribution of foods (FAO 2012).

As an example of this, consider the banana varieties Musa acuminata Colla $\mathrm{x}$ Musa balbisiana Colla, both available in the principal food composition table used in Brazil, the Tabela de Composição de Alimentos (TACO/Unicamp). Considering the data from this source, we find $32 \%$ more potassium in banana-figo than in banana-maçã; we also find $62 \%$ more vitamin $\mathrm{C}$ in banana-da-terra than in bananananica. The genetic diversity of foods affects nutrient profiles. In the past, generic food composition data were considered sufficient for most research purposes, but now the need for cultivar-specific composition data is increasingly being acknowledged (Toledo and Burlingame 2006). When nutrient contents are significantly different in varieties of the same species, the foods should be reported independently in food composition databases and in other printed materials (such as food labels), presenting their unique nutrient profiles (Burlingame et al. 2009). The information should also be shared with the community in health and agriculture programs and food and nutrition education initiatives and incorporated as well into national health and agriculture policies. 
Food composition also varies with agro-ecological zone (soil, climate, management techniques) and seasonality (Hunter et al. 2019). For example, some studies show that foods grown in agro-ecological or organic systems present higher absolute levels of micronutrients (Hunter et al. 2011) and bioactive compounds, such as polyphenols, phenolic acids, isoflavones, stilbene, and anthocyanins (Barański et al. 2014).

The lack of food composition data for UFP is another challenge. Recently, we performed a systematic review of biodiverse food plants in the Caatinga (Jacob et al. 2020), and from 15 studies, we built a list of 65 plants. To date, of these 65 plants, only 3 have accessible nutritional data in the Brazilian Food Composition Table-TACO. We have data for another four in the Tabela Brasileira de Composição de Alimentos (TBCA/USP), and the nutritional data of six other plants are available at the Information System about the Brazilian Biodiversity (SiBBr, in Portuguese). Yet we still lack nutritional data for $80 \%$ of the biodiverse food plants consumed by people in Caatinga.

One explanation for this lack of information is the cost of generating food composition data. During dietary assessment analysis, the process of determining the nutritional value of biodiverse food plants not covered in national food tables commences with a literature review and continues with laboratory studies to generate new data (Nesbitt et al. 2010). We recommend defining priorities and securing reliable identification of food plants before performing the analysis. Food matching strategies that relate data available in the food composition table and other sources lacking food data are an option in cases where proper analysis is not available (FAO 2012).

In addition to these challenges as related to biodiversity in dietary assessments, we bring others. For example, food systems in peri(urban) areas tend to have a higher degree of complexity than in rural areas, considering both (1) the intricate supply chains of retail outlets and urban markets and (2) the higher contribution of processed foods to diets (Lachat et al. 2018). These represent real challenges to mapping biodiverse food chains and to developing the dietary assessment itself. Another difficulty is how to embed this complexity into methods and research presuppositions. We already know that there is a relationship between the number of species consumed (species richness, a biodiversity indicator) and the quality of the diet (mean adequacy ratio, a nutritional indicator) (Lachat et al. 2018). However, we still need to better understand how this diversity translates into health indicators, especially in urban areas, where the dietary matrix changes due to the significant contribution of industrially processed foods. How do these foods interfere with the bioavailability of nutrients in biodiverse foods? We do not as yet know.

The fact is that we need methods of dietary assessment that address the complex set of continuous interrelated variables involved in epidemiological investigations (Sampson 1985; Beaton et al. 1997; Willett 2013; Mumu et al. 2020). Thus, methods normally recognized as the gold standard in clinical research, such as randomized controlled trials (RCT), are not the most suitable to evaluate the effect of dietary intake in epidemiological nutritional outcomes (Zeilstra et al. 2018). For example, 
one of RCT's presuppositions is uniformity, which in nutrition research in general is difficult to achieve. Consider that the composition of foodstuffs can vary substantially across the research setting, and consider the effect of population nonuniformity (where the non-uniformity might also be unknown). Complex interactions influence nutrient consumption and thus physiological exposure. Bad assumptions can lead to ambiguity in similar experiments, yielding results that are nonreproducible. Here, we are not discussing the RCT method. However, we can see that in nutritional research, the critical step is to verify whether a presupposition can be considered valid. We must understand both the culture and environment as being critical factors for food biodiversity analysis and as potential modifiers of the inquiry's presuppositions.

\section{Assessing Biodiverse Food Plants in Dietary Intake Surveys: How to Do It?}

The United Nations Food and Agriculture Organization recently released guidelines to assess biodiverse foods in dietary surveys. In Fig. 2, the flowchart summarizes the main steps for dietary assessment with a focus on food biodiversity. In this section, we will address certain critical steps for this task: elaboration of a tool to support dietary inquiries, generally a photo book (step 6), and the selection and adaptation of a dietary intake instrument (steps 7-9).

To elaborate a photo book, the main recommendations are (1) build a multidisciplinary team, composed of an anthropologist, a nutritionist, food composition experts, and a photographer; (2) analyze food intake patterns, nutritional problems in the studied population, and the available biodiversity; (3) define which biodiverse foods to include in the survey; and (4) ensure correct identification of the biodiverse food plants. The final food list in the dietary assessment tool must combine all foods and varieties of interest and all of the different local food names used. With the food list, vernacular names, correct scientific identification, and photos in hand, we have the material to start production of the photo book.

One of us recently developed a photo book to support a dietary assessment in a Caatinga community (the process is summarized in Fig. 3). We started by building a plant list with plants of interest in the semiarid region, which is mainly in the Caatinga Brazilian biome. We performed a systematic review of biodiverse food plants in the region (Jacob et al. 2020), focusing on UFP. To include conventional plants, we consulted data from national research concerning food consumption (Instituto Brasileiro de Geografia e Estatística 2011). And finally, to gather data on agriculture biodiversity, we visited some local markets. With a plant list ready, together with botanists and photographers, we selected photos. At this stage, we only included photos already identified by specialists, and we also performed two additional identification processes, one with local experts and another with two independent botanists to ensure the accuracy of the images. Our final plant list 


\section{Biodiversity assessment in food consumption studies}

How to do it?

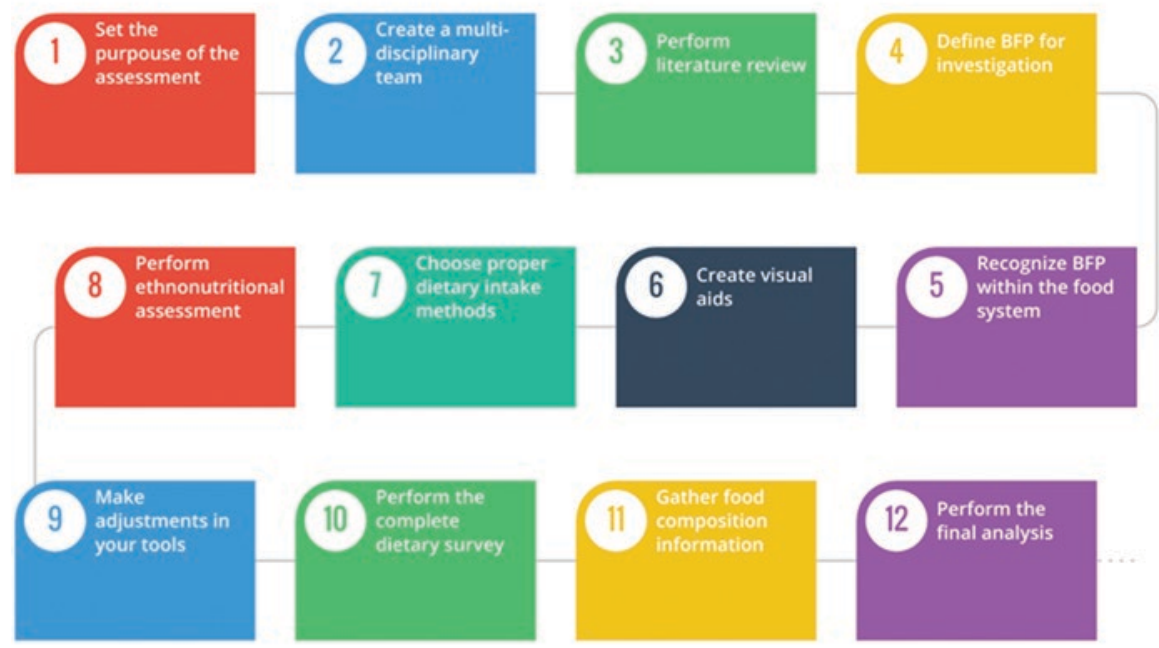

Fig. 2 How to assess biodiverse food plants in dietary intake surveys? The flowchart summarizes the main steps for dietary assessments with a focus on food biodiversity. (Adapted from FAO (2017))

contained 135 items. We chose a minimal design for our photo book, with the plants organized by their vernacular names to easily register the data during the interviews. With this first version in hands, we went to the field to test our tool in an ethnonutrition assessment.

Through mapping of the biodiverse foods available in our study scenario, we started with a list of 135 plants and yet finished the ethnonutrition assessment with 156 plants. The new plants added were mostly agricultural varieties of beans, manioc, orange, mango, and corn (for correct identification of plants, see Nesbitt et al. 2010). We did not include new UFP species since new species were not mentioned in the assessment. On the contrary, we perceived that the community might well be facing lessened UFP consumption, with an increase in highly processed foods. Further, with the community's feedback, we changed certain pictures (giving greater attention to botanical identification keys such as flowers and fruits), and we updated vernacular names to the more common names used in the community. For example, when we asked if people consumed cumaru (Amburana cearensis (Allemão) A.C.Sm.), they told they did not, because they only recognized this plant, the same plant, as amburana-açu. Such vernacular name adjustments were more significant for agricultural varieties than in other plant species. For example, banana-da-terra (Musa acuminata Colla x Musa balbisiana Colla "terra" (AAB)), one kind of banana reported in the Brazilian national Pesquisa de Orçamentos Familiares, is 


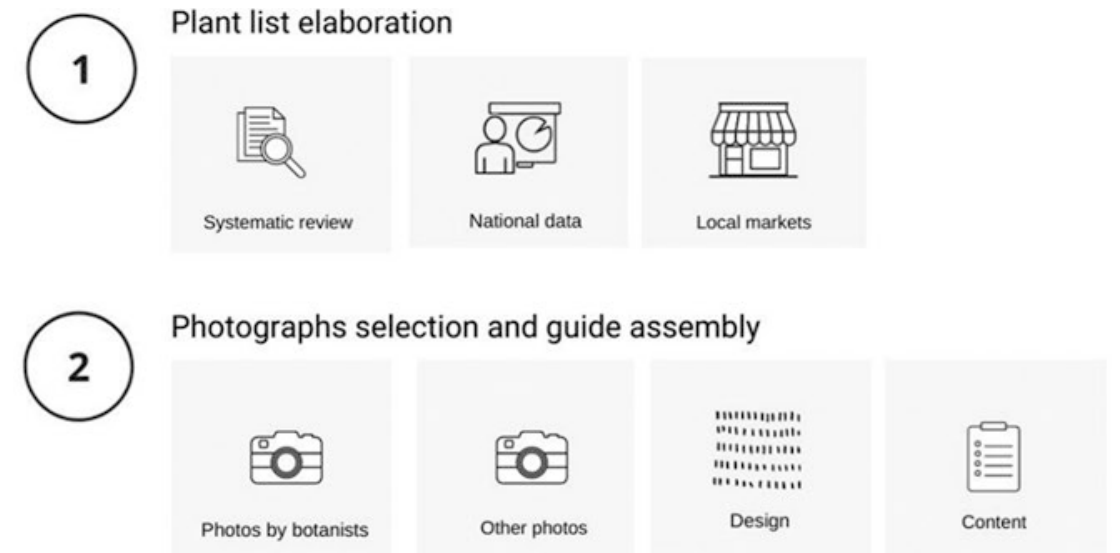

\section{3}

4

\section{5}

\section{Photographic guide assessment by local experts}

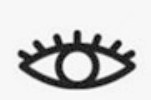

Image recognition

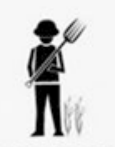

Vernacular names

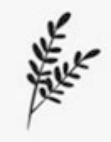

Absent species

\section{Photographic guide corrections}

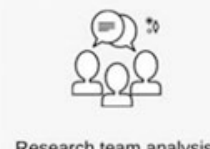

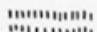

ำi...........

m.............

Research team analysis

Design

\section{Photographic guide assessment by botanists}

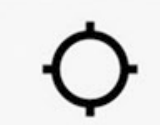

Taxonomic accuracy

\section{Photographic guide final corrections}

\section{6}

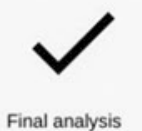

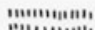

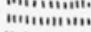

……..........

Final analysis

Design

Fig. 3 Steps to prepare a photographic guide of locally available biodiverse foods. The final product, the photo book with images and names of food resources, is a supportive tool that assists in accurate identification of foods informed during dietary intake surveys. (Source: Authors' elaboration) 
known locally not as banana-da-terra but as banana-comprida. Thus, after the ethnonutritional assessment, we had a more precise guide (quality of pictures, varieties of food, and proper ethnotaxonomy) to proceed with the dietary inquiry. In the case of Brazil, even considering the complexity of populational dietary assessments in urban settings, we suggest that a similar approach can be applied to samples in all Brazilian biomes.

Another step during dietary assessments focusing on biodiversity is selection and adaptation of a dietary intake instrument. A common approach used in extensive epidemiologic studies has been the application of two instruments: the Food Frequency Questionnaire (FFQ) and the 24-hour Dietary Recall (applied to a subsample to evaluate and calibrate the FFQ). Calibration consists of adjusting the FFQ to be more like the Recall results and thus (presumably) be less biased. Another example is administering one or more 24-hour Dietary Recall instruments to the full sample, as a principal instrument in combination with the FFQ, a tool that better sheds light on foods consumed less frequently, such as seasonal plants and meals (Thompson et al. 2015).

We can describe a population group's usual intake with the 24-hour Dietary Recall instrument if we apply it more than once. Since it is an open method, it is effective in cross-cultural contexts, allowing the participant to name a mealtime, a food item, or a local recipe (FAO 2017). Nevertheless, this method has weaknesses. It has a low capacity to recognize food patterns over time. For example, if a food item is sparsely eaten, there is a high chance that the item will not be cited if the survey is performed only once or twice. Many survey days might be needed to register the consumption of certain items, making the research costly and increasing the participant's burden. ${ }^{1}$ On the other hand, a traditional FFQ, being popular in epidemiological studies, relies on a finite list (covering 80-90\% of nutrient intake) of research interest (Mota et al. 2019). The FFQ is used to rank nutrient intake, biocomponents, groups, or food group present in the diet.

Another recommendation for food intake surveys it is to combine the 24-hour Dietary Recall with a Food Propensity Questionnaire (FPQ), with the aid of a photo album or including photos in the platform used to collect the dietary intake data. The FPQ aims to complement information from the 24-hour Dietary Recall instrument to minimize biases related to daily variations in food consumption, which are present if we obtain only a single observation, or the observations are made in short intervals, affecting estimated habitual consumption (Subar et al. 2006).

Finally, we note the process of adapting tools. Adapting 24-hour Dietary Recall involves adding two new columns: one to accurately identify the biodiverse food mentioned by the interviewee and another to match each plant identified with its code in the photo book (FAO 2017). In the case of the FPQ, researchers should use the plant list as elaborated in the photo book development process.

One of us had the opportunity to test both the 24-hour Dietary Recall and FFQ instruments in an ethno-national assessment in a Caatinga community. After testing

\footnotetext{
${ }^{1}$ Currently, the 24-hour Dietary Recall instrument is supported by digital platforms, with an ample (but not exhaustive) list of foods.
} 
these tools in the field, we decided to add two new columns to the FFQ. The first one concerned seasonality (yes or no). We added it to quickly identify seasonal plants during interviews and to accurately convert the consumption frequency for dietary analysis. We also added a column to identify plants that act as food and medicine at the same time. In our analysis, the participants presented to us certain plants of medicinal value (e.g., jatobá, Hymenaea courbaril L.) that they consumed as a tea at breakfast or dinner, even if not being sick, which characterizes a food medicine continuum (Ferreira-Júnior et al. 2018). This (non-binary) system of food classification is different from ours, and our first instinct in the field was to exclude such plants from our food plant list, due to our own biases. However, we re-analyzed our wrong presuppositions and decided to adapt the FFQ. We added a new column to register every plant that possessed a local non-binary identity (food medicine continuum, yes or no). This measure allowed us to identify natural resources that act as both food and medicine. It also prevented undesirable dietary interpretations that result from excluding plants that might have a nutritional impact on the diet. Depending on each investigation's purposes, the research team may find it necessary to develop new adaptations. In every case, we highly recommend previous ethnonutrition assessment of instruments before use in dietary assessment at the populational level.

\section{Fundamental Actions to Mainstream Biodiverse Food Plants in Dietary Assessments}

At this point, it is clear that mainstreaming biodiverse food plants in dietary assessments involves several challenges. How to overcome them? Jacob and Albuquerque (2020) enumerate four main gaps that may help us to design an interdisciplinary research agenda for nutritionists, ethnobiologists, anthropologists, and other scientists to consider biodiverse food resources and the cultural and environmental factors attached to them in our dietary assessments.

The first gap is the lack of accessible food biodiversity data. Brazil has an estimated flora of 48,771 species, including algae, angiosperms, bryophytes, fungi, gymnosperms, ferns, and lycophytes (Instituto de Pesquisas Jardim Botânico do Rio de Janeiro 2020), and we still do not know much about the edible potential of this flora. Due to the accelerating process of biodiversity loss that the world faces (Bongaarts 2019), we must act quickly. The second gap is the lack of culinary data in ethnobiology studies. Ethnobiology studies offer nutrition science-relevant data on food biodiversity and its impact on local food systems (Kuhnlein 2014). However, many studies in this area fail to present how food is prepared before being eaten, which we call ethno-culinary data. Actions such as washing, soaking, and peeling, to mill, to heat, to roast, to boil, to infuse, to germinate, to ferment, to cure, to preserve, and to dehydrate, or combinations of these increase nutrient bioavailability and inactivate or reduce anti-nutritional factors. These need to be collected. 
Without such registration, we cannot use ethnobiological data to determine whether higher biodiversity consumption correlates with better quality diets and food and nutrition security. The third gap is the scarcity of nutritional data, which we already explored in this chapter. Finally, the authors highlight the need for interdisciplinary methods and interprofessional research teams.

We see the cross-disciplinary gap mentioned by the authors as a starting point for action. Cross-disciplinary approaches favor construction of methods that resonate with the dialectic between nature and culture in food plants. Classic works by nutritionists such as Harriet Kuhnlein (1991), biologists such as Nina Etkin (2006), and anthropologists such as Audrey Richards (1948) remind us that high-level scientific dietary assessment research is not a matter of a single science. Multidisciplinary academic efforts must begin in the undergraduate years (FAO 2014). Nutrition students must think about food as more than just nutrients but as landscapes, as biodiversity, and as cultural products. Ethnobiologists must think about dietary processing and diets. Anthropologists must think about nature, diets, and ecology. Agronomists must think about traditional systems and how their decision-making as professionals affects people and nature (Powell et al. 2015). All of these professionals are invited to understand the complex biocultural factors of diet and to embrace food systems thinking (IPES-Food 2015). Professors need to face the contemporary challenge of fragmentation in science and create the space for interprofessional action to advance biodiverse food plant science.

Finally, we wish to highlight the window of opportunity that "Citizen Science" opens for us to gather data on biodiverse food and its associated traditional knowledge. Citizens can participate actively in the advances and discoveries of science. This participation should be informed, conscious, and voluntary. Some steps toward success in this endeavor will be (1) the enhancement of tools for gathering and processing data, (2) the appreciation of projects with real effects in people's lives, and (3) the creation of research consortiums (Bonney et al. 2014; Heigl et al. 2019). To win the respect of participants, scientists, and policymakers, citizen-based science projects need to be responsibly planned and executed with precision. One example of how this might happen is the CONECT-e initiative, ${ }^{2}$ an interactive platform, in wiki format, where people can consult and provide traditional knowledge as related to plants, animals, fungi, and traditional species varieties. The primary mission of CONECT-e in Spain is to preserve and spread traditional ecological knowledge. Similar approaches to map food biodiversity, using participatory methodologies with the support of family farmers, rural communities, and others, can be applied. For instance, the use of geographic information (Fast and Rinner 2018), volunteered from crowd-mapping services, might well be an appropriate solution. ${ }^{3}$ We see citizen science as strategic, because it offers new approaches to realize the biodiverse food plant research agenda.

\footnotetext{
${ }^{2}$ See the CONECT-e platform at https://conecte.es/

${ }^{3}$ See, for example, this experience to map food markets using community participation in a municipality from Brazil: www.nutrir.com.vc/mapa
} 


\section{Conclusion}

In this chapter, we offered various reflections involved in the effort to include cultural and environment variables in nutritional analyses and a set of methods and tools to do so in the field of food consumption studies. We are conscious of the enormous challenges to application in research practice, principally in urban settings and in populational studies. However, we are also aware of how urgently we need to act. The ongoing pandemic outbreak of COVID-19 reinforces the necessity of integrating nutrition research with the environmental challenges imposed by food systems. Within the ecosystem, the nutritional environment is not separate from the biological environment. In other words, human health and environmental health are intrinsically connected, a fact we cannot ignore.

\section{References}

Barański M, Rednicka-Tober D, Volakakis N et al (2014) Higher antioxidant and lower cadmium concentrations and lower incidence of pesticide residues in organically grown crops: a systematic literature review and meta-analyses. Br J Nutr 112:794-811

Beaton GH, Burema J, Ritenbaugh C (1997) Errors in the interpretation of dietary assessments. Am J Clin Nutr 65. https://doi.org/10.1093/ajen/65.4.1100S

Bongaarts J (2019) IPBES, 2019, Summary for policymakers of the global assessment report on biodiversity and ecosystem services of the Intergovernmental Science-Policy Platform on Biodiversity and Ecosystem Services. Popul Dev Rev. https://doi.org/10.1111/padr.12283

Bonney R, Shirk JL, Phillips TB et al (2014) Next steps for citizen science. Science 343:14361437. https://doi.org/10.1126/science. 1251554

Burlingame B, Charrondiere R, Mouille B (2009) Food composition is fundamental to the crosscutting initiative on biodiversity for food and nutrition. J Food Compos Anal 22:361-365. https://doi.org/10.1016/j.jfca.2009.05.003

Ceagesp (2020) Ceagesp Guide. http://www.ceagesp.gov.br/guia-ceagesp. Accessed 1 Aug 2020

de Garine I (1972) The socio-cultural aspects of nutrition. Ecol Food Nutr 1:143-163. https://doi. org/10.1080/03670244.1972.9990282

Etkin N (2006) Edible medicines: an ethnopharmacology of food. The University of Arizona Press, Tucson

FAO (2010) Second report on the state of the world's plant genetic resources for food and agriculture. FAO, Rome

FAO (2012) INFOODS: guidelines for food matching 1.2. FAO, Rome

FAO (2014) Second international conference on nutrition. FAO, Rome

FAO (2017) Guidelines on assessing biodiverse foods in dietary intake surveys. FAO, Rome

FAO (2018) Dietary assessment: a resource guide to method selection and application in low resource settings. FAO, Rome

Fast V, Rinner C (2018) Toward a participatory VGI methodology: crowdsourcing information on regional food assets. Int J Geogr Inf Sci 32:2209-2224. https://doi.org/10.1080/136588 16.2018.1480784

Ferreira-Júnior W, Campos L, Pieroni A, Albuquerque U (2018) Bases biológicas e culturais do uso de plantas alimentícias e medicinais. In: Albuquerque UP (ed) Etnobiologia: bases ecológicas e evolutivas, 2nd edn. NUPEEA, Recife, pp 173-188

Grant G, McKenzie N, Moreira R, Pusztai A (1986) Dioclea grandiflora and Dioclea sclerocarpa seeds. A nutritional study. Qual Plant Plant Foods Hum Nutr 36:47-61 
Heigl F, Kieslinger B, Paul KT et al (2019) Toward an international definition of citizen science. Proc Natl Acad Sci U S A 116:8089-8092. https://doi.org/10.1073/pnas.1903393116

Herforth A, Ahmed S (2015) The food environment, its effects on dietary consumption, and potential for measurement within agriculture-nutrition interventions. Food Sec 7:505-520

Hunter D, Foster M, Mcarthur JO et al (2011) Evaluation of the micronutrient composition of plant foods produced by organic and conventional agricultural methods. Crit Rev Food Sci Nutr 51:571-582. https://doi.org/10.1080/10408391003721701

Hunter D, Borelli T, Beltrame DMO et al (2019) The potential of neglected and underutilized species for improving diets and nutrition. Planta 250:709-729. https://doi.org/10.1007/ s00425-019-03169-4

Instituto Brasileiro de Geografia e Estatística (2011) Pesquisa de orçamentos familiares 20082009: análise do consumo alimentar pessoal no Brasil. Brasília

Instituto de Pesquisas Jardim Botânico do Rio de Janeiro (2020) Flora do Brasil 2020 - Algas, Fungos e Plantas. http://reflora.jbrj.gov.br/. Accessed 1 Dec 2019

IPES-Food (2015) The new science of sustainable food systems. Belgium

Jacob M, Albuquerque U (2020) Biodiverse food plants: Which gaps do we need to address to promote sustainable diets? Ethnobiol Conserv 9:1-7. https://doi.org/10.15451/ec2020

Jacob M, Medeiros M, Albuquerque U (2020) Biodiverse food plants in the semiarid region of Brazil have unknown potential: a systematic review. PLoS One. https://doi.org/10.1371/journal.pone.0230936

Khoury CK, Bjorkman AD, Dempewolf $\mathrm{H}$ et al (2014) Increasing homogeneity in global food supplies and the implications for food security. Proc Natl Acad Sci U S A 111:4001-4006. https:// doi.org/10.1073/pnas.1313490111

Kuhnlein H (1991) Traditional plant foods of Canadian indigenous peoples: nutrition, botany and use. Gordon and Breach Publishers, Quebec

Kuhnlein HV (2014) How ethnobiology can contribute to food security. J Ethnobiol 34:12-27. https://doi.org/10.2993/0278-0771-34.1.12

Lachat C, Raneri JE, Smith KW et al (2018) Dietary species richness as a measure of food biodiversity and nutritional quality of diets. Proc Natl Acad Sci 115:127-132. https://doi.org/10.1073/ pnas. 1709194115

Mota V, Lima S, Lyra C (2019) Questionário de Frequência Alimentar. In: Marchioni DML, Gorgulho BM, Steluti J (eds) Consumo Alimentar: guia para avaliação. Manole, São Paulo, pp 8-23

Mumu SJ, Merom D, Ali L et al (2020) Validation of a food frequency questionnaire as a tool for assessing dietary intake in cardiovascular disease research and surveillance in Bangladesh. Nutr J 19. https://doi.org/10.1186/s12937-020-00563-7

Nesbitt M, Mcburney RPH, Broin M, Beentje HJ (2010) Linking biodiversity, food and nutrition: the importance of plant identification and nomenclature. J Food Compos Anal 23:486-498. https://doi.org/10.1016/j.jfca.2009.03.001

Powell B, Thilsted SH, Ickowitz A et al (2015) Improving diets with wild and cultivated biodiversity from across the landscape. Food Sec 7:535-554. https://doi.org/10.1007/s12571-0150466-5

Richards A (1948) Hunger and work in a savage tribe. Greenwood Press, Westport

Ruel MT, Alderman H (2013) Nutrition-sensitive interventions and programmes: how can they help to accelerate progress in improving maternal and child nutrition? Lancet 382:536-551. https://doi.org/10.1016/S0140-6736(13)60843-0

Sampson L (1985) Food frequency questionnaire as a research instrument. Clin Nutr 4:171-178

Subar AF, Dodd KW, Guenther PM et al (2006) The food propensity questionnaire: concept, development, and validation for use as a covariate in a model to estimate usual food intake. J Am Diet Assoc 106:1556-1563. https://doi.org/10.1016/j.jada.2006.07.002

Thompson FE, Kirkpatrick SI, Subar AF et al (2015) The National Cancer Institute's dietary assessment primer: a resource for diet research. J Acad Nutr Diet 115:1986-1995. https://doi. org/10.1016/j.jand.2015.08.016 
Toledo A, Burlingame B (2006) Biodiversity and nutrition: a common path toward global food security and sustainable development. J Food Compos Anal 19:477-483

Tumilowicz A, Neufeld L, Pelto GH (2016) Using ethnography in implementation research to improve nutrition interventions in populations. Matern Child Nutr 11:55-72

Willett W (2013) Nutritional epidemiology, 3rd edn. Oxford University Press, New York

Willett W, Rockström J, Loken B et al (2019) Food in the Anthropocene: the EAT-Lancet Commission on healthy diets from sustainable food systems. Lancet 393:447-492. https://doi. org/10.1016/S0140-6736(18)31788-4

Zeilstra D, Younes JA, Brummer RJ, Kleerebezem M (2018) Fundamental limitations of the randomized controlled trial method in nutritional research: the example of probiotics. Adv Nutr. https://doi.org/10.1093/ADVANCES/NMY046

Zimmerer KS (2015) Understanding agrobiodiversity and the rise of resilience: analytic category, conceptual boundary object or meta-level transition? Resilience. https://doi.org/10.1080/2169 3293.2015.1072311 